\title{
Openness, Transparency and Equity in Public Health Surveillance Data Sharing
}

\author{
Matthew Brack*, Michael Edelstein, Asha Herten-Crabb and David R. Harper \\ ${ }^{1}$ Centre on Global Health Security, Chatham House, London, United Kingdom;
}

\section{Objective}

To address both the policy and technical issues of sharing public health surveillance data across national borders with the aim of establishing new norms so that data can be shared in an open, transparent and equitable way.

\section{Introduction}

The outbreaks of Severe Acute Respiratory Syndrome (SARS) in 2003, influenza A (H1N1) in 2009 and Ebola in 2014 have shown increasingly that infectious diseases can spread globally in a short timeframe, affecting both high- and low-income countries. Taking action to mitigate the impact of future crises relies on sharing public health surveillance data across national borders in an efficient and effective way. However, data users, particularly in high-income countries, often use surveillance data, particularly from low- and middle-income countries, with little or no benefit to the data generator. As Indonesia's refusal to share influenza virus sequences during the 2006 H5N1 outbreak illustrates, this imbalance increases reluctance to share and jeopardizes the global good that can be achieved. In order to share public health surveillance data internationally in an equitable way, technical, political, ethical, and legal issues need to be addressed. The Centre on Global Health Security at Chatham House is producing guidance that will address both the policy and technical issues with the aim of establishing new norms so that data can be shared in an open, transparent and equitable way.

\section{Methods}

We have developed key principles on the technical, legal, ethical, and political implications of cross-border data sharing. These draw on the published literature and expert advice gained through interviews and a series of thematic roundtables.

\section{Results}

Open, transparent and equitable public health surveillance data sharing requires the engagement of three groups of stakeholders: those generating the data; those who interpret data generated by others; and those facilitating the data-sharing process. These categories are not mutually exclusive. We have outlined seven key principles that encourage optimal global public health surveillance data sharing and promote the equal distribution of benefits: (i) articulating the value proposition; (ii) planning for data sharing; (iii) ensuring high-quality data production; (iv) collaborating in creating data-sharing agreements; (v) building trust and being consistent; (vi) understanding the global legal landscape; and (vii) monitoring and evaluating progress. In addition, standards, capacity building, and ethical considerations, such as those concerning equity, are themes that span these principles and have to be embedded in each of them.

\section{Conclusions}

Surveillance is a cornerstone of public health. Increasingly, public health surveillance data need to cross national borders to address international public health crises - the traditional health security approach of protecting national borders clearly does not work in a globalized world. The Ebola crisis in West Africa has demonstrated that a local event in a remote location can have widespread consequences, and that global health security is only as strong as its weakest point. Cross-border public health surveillance data that are both high quality and timely are essential in supporting efforts to mitigate the impact of such crises. The Chatham House guidance, scheduled for launch in October 2016, is expected to play a key role in the global community's management of future threats.

\section{Keywords}

data sharing; surveillance data; equity; International

*Matthew Brack

E-mail:mbrack@chathamhouse.org 